\title{
Sintomatología física y mental asociada al síndrome de burnout en los profesionales de la enseñanza
}

\author{
Jesús Esteras, Paloma Chorot y Bonifacio Sandín \\ Universidad Nacional de Educación a Distancia, Madrid, España
}

\begin{abstract}
Physical and mental symptomatology associated with burnout syndrome in teachers. The aim of the present study was to examine the association between burnout and somatic and psychopathological symptomatology, in a sample of 171 teachers working in educational institutions (preschool, elementary school, and middle school). Participants completed the Maslach Burnout Inventory (MBI), the Somatic Symptoms Scale-Revised (Escala de Síntomas Somáticos-Revisada, ESS-R), and the Symptoms Assessment-45 Questionnaire (SA-45). Results indicate that the MBI dimension of «emotional exhaustion» is closely associated with somatic and psychopathological symptoms. Also, we found that teachers with burnout syndrome reported a higher level of somatic and psychopathologic symptomatology compared to non-burnout teachers. Results are discussed in relation to possible clinical profiles associated with burnout.
\end{abstract}

Key words: Burnout syndrome; teachers; somatic symptoms; psychopathological symptoms, health.

Resumen: El objetivo del presente estudio consistió en examinar la asociación entre el burnout y la sintomatología somática y psicopatológica en una muestra de 171 docentes que trabajaban en centros educativos (preescolar, primaria, y ESO/bachillerato). Los participantes cumplimentaron el Maslach Burnout Inventory (MBI), la Escala de Síntomas Somáticos-Revisada (ESS-R), y el Symptoms Assessment-45 Questionnaire (SA-45). Los resultados indican que la dimensión de «agotamiento emocional» del MBI se asocia estrechamente a la sintomatología somática y psicopatológica. Así mismo, se constata que los docentes con síndrome de burnout informan de mayor nivel de sintomatología somática y psicopatológica comparado con los docentes sin el síndrome. Se discuten los resultados en relación con los posibles perfiles clínicos asociados al burnout.

Palabras clave: Síndrome de burnout; docentes; profesores; síntomas somáticos; síntomas psicopatológicos; salud.

\section{Introducción}

El concepto de «burnout» ha adquirido gran popularidad especialmente a partir del modelo tripartito de Maslach (p.ej., Maslach y Jackson, 1981). Esta autora definió el burnout como un síndrome que ocurre entre individuos que trabajan con personas, y se caracteriza por tres dimensiones fundamentales: (a) agotamiento emocional, (b) despersonalización (i.e., actitudes y sentimientos cínicos sobre los clientes), y (c) baja realización personal (Esteras, Chorot y Sandín, 2018; Seisdedos, 1997); aunque no todas las dimensiones parecen

Recibido: 3 de junio 2018: aceptado: 3 de septiembre 2018 Correspondencia: Paloma Chorot, Universidad Nacional de Educación a Distancia, Facultad de Psicología, Juan del Rosal 10, 28040 Madrid, España. Correo-e: pchorot@psi.uned.es. tener la misma relevancia (Montero-Marín et al., 2016). Algunos autores, no obstante, han resaltado el papel de la dimensión de agotamiento emocional, ya que podría entenderse como «un estado de agotamiento físico, emocional y mental causado por una implicación temporalmente prolongada en situaciones de elevada demanda emocional (Sandín, 2008).

La evidencia de la literatura suele indicar que existe cierta asociación entre el síndrome de burnout y el grado percibido de bienestar o salud (Aluja, 1997; Ballester-Arnal et al., 2016; Esteras, Chorot y Sandín, 2018; García, 1991; García, Sáez y Llor, 2000; Santamaría y Soto, 2005), así como también con los niveles de ansiedad y depresión (Firth, McKeown, Mcintee y Britton, 1987; Esteras et al., 2018; García et al., 2000. Aunque los estudios relacionados con el ámbito de la docencia son escasos, algunos trabajos han encontrado una rela- 
ción significativa entre el síndrome de burnout y los problemas de salud en profesionales de la enseñanza (Capone, Joshanloo y Park, 2019; Durán, Extremera, Montalbán y Rey, 2005; Holt, Fine y Tollefton, 1987; Greenglass y Burke, 1988). Estos estudios han sugerido que el síndrome de burnout parece asociarse a diversos tipos de alteraciones físicas y emocionales (ansiedad y depresión).

No obstante, en línea con lo señalado por Sandín (2008), la dimensión de agotamiento emocional parece asociarse de forma más estrecha con la perturbación de la salud en comparación con las dos restantes dimensiones del burnout (despersonalización y baja realización personal) (Calvete y Villa, 2000; Fernández, 2000; Gil-Monte, Valcáercer, Grau y Peiró, 1996). En este sentido, el agotamiento emocional se ha relacionado con la depresión, los trastornos obsesivo-compulsivos, la ansiedad, la hostilidad y la ideación paranoide. También se ha sugerido evidencia a favor de un posible papel de la dimensión de la despersonalización en la sintomatología de tipo psicótico (ideación paranoide, personalidad psicótica, y hostilidad) (Cáceres, 2006).

Aunque la evidencia de la literatura sobre el burnout es amplia, los estudios sobre la implicación del burnout en el ámbito de la docencia son escasamente concluyentes, especialmente en lo que concierne a su relación con signos o síntomas de salud (física y/o mental). De hecho, aunque los estudios sobre el burnout en sí mismos son muy extensos, son escasos los que asocian el burnout con otras variables de salud mental. El clásico estudio de revisión teórica y metodológica sobre estrés laboral, burnout y salud en docentes (Guglielmi y Tatrow, 1998) sugiere que, aunque la literatura generalmente tiende a apoyar la hipótesis de que el estrés ocupacional asociado a la docencia se asocia a empobrecimiento de la salud en los profesores, tal conclusión no es consistente. A nuestro juicio, algunos de los problemas metodológicos tienen que ver con la no diferenciación entre la conceptualización dimensional del burnout (i.e., mediante los diferentes componentes) y la conceptualización categorial (i.e., tener o no tener síndrome de burnout); y porque para el diagnóstico categorial no existe un único criterio aceptado por la comunidad científica.

Mediante el presente estudio hemos pretendido examinar la relación entre el burnout y la salud física y mental desde ambas ópticas del concepto de burnout, i.e., desde su concepción dimensional basada en las tres dimensiones clásicas del burnout (agotamiento emocional, despersonalización, y realización personal) y desde el enfoque categorial (presencia o no del síndrome de burnout). El diagnóstico del síndrome de burnout se estableció de acuerdo con el criterio de Seisdedos (1997), según el cual los sujetos con síndrome de burnout serían aquellos que muestren elevadas puntuaciones en agotamiento emocional y en despersonalización, y bajas puntuaciones en realización personal (para más detalle, véase la descripción del MBI en la sección de Instrumentos). La sintomatología física y psicopatológica se evaluó a través de instrumentos de amplio espectro, considerando los principales sistemas somatofisiológicos (para la evaluación de los síntomas físicos) y de síntomas psicopatológicos (para la evaluación de la salud mental). Ambos aspectos son fundamentales para una visión comprensiva sobre las características psicopatológicas y psicosomáticas asociadas al burnout.

Esperamos encontrar asociaciones positivas y significativas entre las tres dimensiones de burnout y los síntomas físicos y mentales, aunque, de acuerdo con la evidencia de la literatura, hipotetizamos una relación más estrecha con la dimensión de agotamiento emocional que con las dos restantes dimensiones de burnout. Así mismo, esperamos encontrar diferencias significativas entre los individuos con diagnóstico de síndrome de burnout y los individuos sin el síndrome para las diferentes variables de sintomatología física y mental.

\section{Método}

\section{Participantes}

La muestra estuvo constituida por 171 docentes de enseñanzas no universitarios de Benidorm, lo que representaba el $20.12 \%$ de la población total de docentes de esta ciudad (850). De ellos un $72.5 \%$ eran mujeres $(n=124)$ y un $27.5 \%$ varones $(\mathrm{n}=47)$. El rango de edad de la muestra estaba comprendido entre 22 y 64 años (media $=39.23$ años, $D T=11.12)$. La edad media era similar para el grupo de hombres que para el de mujeres $(\mathrm{t}=.99, \mathrm{~ns})$.

La selección de los participantes se llevó a cabo mediante un muestreo estratificado de docentes en el municipio de Benidorm. Se crearon estratos según las zonas del municipio (tres zonas escolares), por etapas educativas (Educación Infantil, Primaria, ESO y Bachillerato) y según el tipo de centro (público, privado concertado y privado no concertado). Así la muestra de docentes debía representar a la totalidad de tipos de centros, zonas geográficas del municipio y etapas educativas.

\section{Instrumentos}

Aparte de un cuestionario que se elaboró específicamente para registrar los datos sociodemográficos (edad, sexo, etapa educativa, etc.), se aplicaron los siguientes instrumentos de evaluación: 
Maslach Burnout Inventory (MBI; Maslach y Jackson, 1981). Se aplicó la versión española validada por Seisdedos (1997). Consta de 22 ítems en forma de afirmaciones sobre los sentimientos, pensamientos y actitudes personales que se experimentan en el trabajo. Se valoran con una escala de tipo Likert, que indica la frecuencia con la que se experimenta cada sentimiento descrito en el ítem, variando entre 0 («nunca») y 6 («todos los días»). Mediante tres escalas permite evaluar las tres dimensiones siguientes de burnout: agotamiento emocional (9 ítems), despersonalización (5 ítems) y realización personal ( 8 ítems). En el presente estudio obtuvimos los siguientes coeficientes alfa para las tres escalas: .86 (agotamiento emocional), .66 (despersonalización) y .81 (realización personal). De acuerdo con Seisdedos (1997), se establecen tres niveles de gravedad de burnout que corresponden a las puntuaciones del tercio inferior (centiles 1 a 33), tercio central (centiles 34 a 36) y tercio superior (centiles 67 a 99). Se considera que una persona presenta síndrome de burnout cuando muestra puntuaciones elevadas (tercio superior) en agotamiento emocional y despersonalización, y puntuaciones bajas (tercio inferior) en realización personal.

Escala de Síntomas Somáticos-Revisada (ESS-R; Sandín y Chorot, 1995; véase Sandín, Valiente y Chorot, 2008). Es un instrumento de autoinforme que evalúa los diferentes tipos de manifestaciones somáticas relacionadas con la salud de la persona. Consta de 90 ítems con una escala de valoración de tipo Likert, que indica la frecuencia con la que se ha experimentado cada síntoma. Dicha escala de frecuencia presenta 5 grados que van de 0 («nunca durante el último año») a 4 («más de una vez al mes»). La escala presenta nueve subescalas: Inmunológico general, cardiovascular, respiratorio, gastrointestinal, neurosensorial, músculo-esquelético, piel-alergia, génito-urinario, y reproductor femenino (exclusiva para las mujeres). Así mismo, puede obtenerse una puntuación global en síntomas somáticos. Según los autores de la versión española, la estabilidad temporal (correlación test-retest) para un periodo de un mes varió de .73 a .82 . Presenta una alta validez predictiva en relación con otros indicadores de salud física (Sandín, Valiente y Chorot, 2008). En el presente estudio la consistencia interna (coeficiente alfa de Cronbach) fue como sigue: .45 para inmunológico general, .83 para cardiovascular, .87 para respiratorio, .84 para gastrointestinal, .82 para neurosensorial, .85 para músculo-esquelético, .86 para piel-alergia, .83 para genito-urinario, y .80 para reproductor femenino.

Symptom Assessment-45 Questionnaire (SA-45; Davison et al., 1997). Se utilizó la versión española va- lidada por Sandín, Valiente, Chorot, Santed y Lostao (2008). Consta de 45 ítems sobre síntomas psicopatológicos que posibilitan la evaluación de las nueve escalas (hostilidad, somatización, depresión, obsesión-compulsión, ansiedad, sensibilidad interpersonal, ansiedad fóbica, ideación paranoide y psicoticismo), cada escala es definida por 5 ítems. El cuestionario se contesta señalando la intensidad con que ha experimentado lo que se indica en cada uno de los ítems durante la última semana. La intensidad se estima según cinco grados que van de 0 («nada en absoluto») a 4 («mucho o extremadamente»). Aparte de las nueve escalas, también puede obtenerse un índice global de gravedad (puntuación global). En el presente estudio la consistencia interna (coeficiente alfa de Cronbach) fue de .86 para la subescala de hostilidad, .85 para la somatización, .83 para depresión, .81 para obsesión-compulsión, .83 para ansiedad, .87 para sensibilidad interpersonal, .85 para ansiedad fóbica, .74 para ideación paranoide, y .71 para psicoticismo.

\section{Procedimiento}

Se invitó a participar en la investigación a la totalidad de colegios públicos y privados del municipio de Benidorm (Alicante, España), informando previamente al equipo directivo de cada centro y posteriormente al profesorado. Participaron los siguientes centros educativos: doce colegios públicos de educación infantil y primaria; un colegio privado concertado de infantil, primaria, ESO y bachillerato; un colegio privado no concertado de infantil, primaria, ESO y bachillerato; y cuatro institutos de educación secundaria públicos (ESO y bachillerato). Colaboraron un total de 18 centros educativos y se obtuvieron un total de 171 protocolos válidos.

\section{Resultados}

Relación entre las dimensiones de burnout y las
dimensiones de síntomas físicos y psicopatológicos

En la Tabla 1 se indican las correlaciones entre las tres dimensiones de burnout y los síntomas físicos. Como cabría esperar, las dos primeras dimensiones correlacionan de forma positiva con la sintomatología física, mientras que la realización personal lo hace de forma negativa. Cabe señalarse, así mismo, que las correlaciones más elevadas se refieren a la dimensión de agotamiento emocional (las correlaciones varían entre $.16 \mathrm{y}$ .41); esta variable de burnout correlaciona de forma significativa con todas las variables de síntomas somáticos 
y con la puntuación total. Aunque las correlaciones en general tienden a ser moderadas, todas ellas son significativas estadísticamente. En contraste, las correlaciones con las dimensiones de despersonalización y realización personal son bajas y menos significativas. Cabe destacarse, no obstante, que cinco dimensiones de síntomas somáticos correlacionan significativamente con despersonalización, y sólo una con realización personal (véase la Tabla 1).

Las correlaciones entre las dimensiones de burnout y las dimensiones de síntomas psicopatológicos adoptan un patrón general similar al indicado para los síntomas físicos, ya que las principales correlaciones se dan con el agotamiento emocional, siendo menos relevantes las correlaciones con la despersonalización y la realización personal (véase la Tabla 2). Como puede apreciarse en esta tabla, todas las correlaciones entre agotamiento emocional y síntomas psicopatológicos (excepto con psicoticismo) fueron significativas, aunque el tamaño del efecto no pasó de ser moderado en las distintas dimensiones sintomatológicas. No obstante, una pequeña diferencia respecto a las correlaciones con la sintomatología física viene dada porque aquí las correlaciones con realización personal parecen resultar ligeramente más relevantes que las correlaciones exhibidas con la dimensión de despersonalización, aunque caben destacarse las correlaciones significativas de esta última dimensión con ideación paranoide y psicoticismo (véanse las Tablas 1 y 2).

Tabla 1. Correlaciones producto-momento entre las variables de burnout (MBI) y las dimensiones de los síntomas somáticos $(\mathrm{ESS}-\mathrm{R})(N=171)$

\begin{tabular}{lccc}
\hline & $\mathrm{AE}$ & $\mathrm{DP}$ & $\mathrm{RP}$ \\
\hline Inmunológico general & $.39 * *$ & $.29^{* *}$ & -.19 \\
Cardiovascular & $.30^{* *}$ & $.17 *$ & .06 \\
Respiratorio & $.27 * *$ & $.17 *$ & -.12 \\
Gastrointestinal & $.29 * *$ & .07 & -.06 \\
Neurosensorial & $.30^{* *}$ & $.24 * *$ & -.12 \\
Músculo-esquelético & $.38^{* *}$ & $.19 *$ & $-.17 *$ \\
Piel-alergia & $.21 * *$ & .15 & -.09 \\
Genito-urinario & $.17 *$ & .14 & -.07 \\
Reproductor femenino & $.16^{*}$ & .04 & -.07 \\
Global síntomas somáticos & $.41^{* *}$ & $.21 * *$ & -.15 \\
\hline
\end{tabular}

Nota: $\mathrm{AE}=$ agotamiento emocional, $\mathrm{DP}=$ despersonalización, $\mathrm{RP}=$ realización personal. ${ }^{*} p<.05 * * p<.01$.
Tabla 2. Correlaciones producto-momento entre las variables de burnout (MBI) y las dimensiones de sintomatología psicopatológica $(\mathrm{SA}-45)(N=171)$

\begin{tabular}{lccc}
\hline & $\mathrm{AE}$ & $\mathrm{DP}$ & $\mathrm{RP}$ \\
\hline Hostilidad & $.16^{*}$ & .06 & $-.17^{*}$ \\
Somatización & $.31^{* *}$ & .12 & -.12 \\
Depresión & $.29^{* *}$ & .13 & $-.19^{*}$ \\
Obsesión-compulsión & $.29^{* *}$ & .15 & -.11 \\
Ansiedad & $.35^{* *}$ & .13 & $-.16^{*}$ \\
Sensibilidad interpersonal & $.20^{*}$ & .10 & -.15 \\
Ansiedad fóbica & $.17^{*}$ & .12 & -.09 \\
Ideación paranoide & $.32^{* *}$ & $.25^{* *}$ & $-.15^{*}$ \\
Psicoticismo & .08 & $.16^{*}$ & .01 \\
IG de estado psicopatológico & $.28^{* *}$ & .15 & -.15 \\
\hline
\end{tabular}

Nota $: \mathrm{AE}=$ agotamiento emocional, DP $=$ despersonalización, $\mathrm{RP}=$ realización personal, $\mathrm{Ig}=$ índice global. ${ }^{*} p<.05 * * p<.01$.

Diferencias en sintomatología somática y psicopatológica en función de la prevalencia del sindrome de burnout

De la muestra total de docentes, 43 (25.1\%) fueron diagnosticados de síndrome de burnout; los 128 restantes $(74.9 \%)$ no poseían síndrome de burnout. A partir de esto se aplicó un diseño de MANOVA de un factor de grupo (síndrome vs. no síndrome) y las 9 dimensiones de síntomas somáticos, controlando el efecto de la edad y el sexo. Constatamos un efecto significativo sobre las variables de síntomas somáticos, en función de la presencia o ausencia del síndrome de burnout (Hotelling, $\left.F_{(10,155)}=3.12, p<.05\right)$.

Posteriormente, aplicando análisis de varianza de un factor (ANOVA), encontramos que las puntuaciones eran significativamente diferentes en todas las dimensiones de los síntomas somáticos en función de la presencia vs. ausencia del síndrome de burnout, a excepción de las dimensiones gastrointestinal, piel-alergia, génito-urinario y reproductor femenino (véase la Tabla 3). Como indicamos en la tabla, las puntuaciones medias son siempre superiores en el grupo con síndrome de burnout.

Puesto que el efecto de la variable sexo resultó ser estadísticamente significativo $\left(F_{(10,155)}=9.95, p<.05\right)$ en el MANOVA, aplicamos diseños de ANOVA de 2 (síndrome sí vs. síndrome no) x 2 (sexo), controlando el efecto de la edad, con objeto de examinar la interacción entre las variables de síndrome de burnout y sexo en relación con las diferencias en sintomatología somática (se aplicaron análisis de varianza univariados para las varia- 
Tabla 3. Datos descriptivos de las dimensiones de síntomas somáticos (ESS-R) y la presencia vs. ausencia del síndrome de burnout

\begin{tabular}{|c|c|c|c|c|}
\hline & $\begin{array}{l}\text { Sin síndrome burnout } \\
\qquad(n=128)\end{array}$ & $\begin{array}{l}\text { Con síndrome burnout } \\
\qquad(n=43)\end{array}$ & & \\
\hline Variables & $M(D T)$ & $M(D T)$ & $F(1,169)$ & $\eta_{p}^{2}$ \\
\hline Inmunológico general & $7.5(4.2)$ & $10.4(5.4)$ & $13.71^{*}$ & .075 \\
\hline Cardiovascular & $4.2(5.3)$ & $6.4(6.6)$ & $4.42 *$ & .025 \\
\hline Respiratorio & $6.9(6.1)$ & $9.7(8.3)$ & $5.69 *$ & .033 \\
\hline Gastrointestinal & $8.8(6.5)$ & $11.1(8.2)$ & 3.40 & .020 \\
\hline Neurosensorial & $4.9(4.5)$ & $8.8(7.5)$ & $16.34^{*}$ & .088 \\
\hline Músculo-esquelético & $9.8(6.4)$ & $14.4(9.0)$ & $13.10^{*}$ & .072 \\
\hline Piel-alergia & $8.2(7.6)$ & $10.7(8.7)$ & 3.20 & .019 \\
\hline Génito-urinario & $4.6(5.0)$ & $6.0(7.3)$ & 1.94 & .011 \\
\hline Reproductor femenino & $7.2(7.6)$ & $9.4(9.3)$ & 2.29 & .014 \\
\hline Global síntomas somáticos & $55.7(35.4)$ & $84.0(52.1)$ & $15.86^{*}$ & .089 \\
\hline
\end{tabular}

Nota: $\eta_{\mathrm{p}}^{2}=\operatorname{eta}^{2}$ parcial (tamaño del efecto). ${ }^{*} p<.05$.

bles de síntomas somáticos y para la puntuación total en sintomatología somática). Los análisis de varianza reflejaron ausencia de efectos significativos en la interacción entre ambas variables (síndrome de burnout $\times$ sexo) sobre cada dimensión de síntomas somáticos. Esto sugiere que el sexo no actúa como moderador del efecto del burnout sobre la sintomatología somática.

Procedimos de forma similar para probar el efecto del síndrome de burnout sobre la sintomatología de tipo psicopatológico; aplicamos los mismos diseños de MANOVA y ANOVA sobre las dimensiones de síntomas psicopatológicos. Tras aplicar el MANOVA, observamos un efecto significativo sobre las variables de síntomas psicopatológicos, (Hotelling, $F_{(10,154)}=2.77, p<.05$ ) en función de la presencia del síndrome de burnout. La covariable sexo también resultó ser estadísticamente significativa $\left(F_{(10,154)}=1.88, p<.05\right)$. Los resultados de los ANOVAs correspondientes a las diferentes dimensiones de síntomas psicopatológicos se indican en la Tabla 4. Puede observarse en la tabla que el efecto del factor grupo resultó ser estadísticamente significativo para todas las variables de síntomas, excepto para las variables de hostilidad, sensibilidad interpersonal, ansiedad fóbica y psicoticismo. Al igual que ocurría con las variables de síntomas somáticos, en todas las variables se aprecia un incremento de la sintomatología asociada al síndrome, aunque no en todos los casos el incremento resultó ser estadísticamente significativo. Finalmente, tras aplicar el diseño de ANOVA de $2 \times 2$ (síndrome de burnout $\times$ sexo), no encontramos efectos significativos de interacción entre el burnout y el sexo, sugiriendo que, como ocurría con la sintomatología somática, esta última va- riable no interviene modulando el efecto del burnout sobe los síntomas psicopatológicos.

\section{Discusión}

El objetivo del presente estudio fue examinar la asociación entre el burnout y la salud física y mental en una muestra de docentes de enseñanzas no universitarias. Más específicamente, deseábamos ver (a) las manifestaciones sintomatológicas asociadas a las dimensiones del síndrome de burnout, y (b) hasta qué punto dichas manifestaciones psicopatológicas variaban en función del diagnóstico de síndrome de burnout.

En relación con el primer objetivo, esperabamos encontrar asociaciones positivas y significativas entre las tres dimensiones de burnout y los síntomas físicos y mentales, aunque, de acuerdo con la evidencia de la literatura, hipotetizamos una relación más estrecha con la dimensión de agotamiento emocional que con las dos restantes dimensiones de burnout. De acuerdo con los datos obtenidos, constatamos que el agotamiento emocional correlacionaba de forma significativa con todas las dimensiones de sintomatología somática, aunque las correlaciones más elevadas corresponden a los síntomas de tipo musculo-esquelético, inmunológico general, cardiovascular, neurosensorial y gastrointestinal, así como también con la puntuación total en la escala de síntomas somáticos. En contraste, las correlaciones de los síntomas con las otras dos dimensiones de burnout (despersonalización y realización personal) son más limitadas, especialmente con la realización personal, con la que apenas aparecen signos de correlación. Parece pues que 
Tabla 4. Datos descriptivos de las dimensiones de síntomas psicopatológicos (SA-45) y la presencia vs. ausencia del síndrome de burnout

\begin{tabular}{lcccc}
\hline \multicolumn{4}{c}{$\begin{array}{c}\text { Sin síndrome burnout } \\
(n=128)\end{array}$} & $\begin{array}{c}\text { Con síndrome burnout } \\
(n=43)\end{array}$ \\
\hline \multicolumn{1}{c}{ Variables } & $M(D T)$ & $M(D T)$ & $F(1,164)$ & $\eta_{p}^{2}$ \\
\hline Hostilidad & $2.0(3.0)$ & $2.8(3.6)$ & 2.13 & .013 \\
Somatización & $4.6(3.9)$ & $6.8(5.5)$ & $7.08^{*}$ & .042 \\
Depresión & $3.5(3.4)$ & $5.3(4.3)$ & $8.48^{*}$ & .049 \\
Obsesión-compulsión & $4.6(3.5)$ & $6.6(4.5)$ & $8.10^{*}$ & .047 \\
Ansiedad & $3.0(3.2)$ & $4.3(4.1)$ & $4.22^{*}$ & .025 \\
Sensibilidad interpersonal & $4.6(4.2)$ & $5.9(4.6)$ & 2.82 & .017 \\
Ansiedad fóbica & $1.8(2.7)$ & $2.7(4.2)$ & 1.81 & .011 \\
Ideación paranoide & $4.5(3.2)$ & $6.7(4.3)$ & $11.34^{*}$ & .065 \\
Psicoticismo & $2.3(3.1)$ & $2.2(2.5)$ & .10 & .001 \\
I.G. estado psicopatológico & $30.4(23.2)$ & $42.1(30.5)$ & $6.37^{*}$ & .038 \\
\hline
\end{tabular}

Nota: $\eta_{\mathrm{p}}^{2}=$ eta $^{2}$ parcial (tamaño del efecto). ${ }^{*} p<.05$.

el agotamiento emocional exhibe una relación con los síntomas somáticos bastante estrecha, lo cual contrasta con las restantes dimensiones de burnout. Estos resultados apoyan la evidencia presentada por otros autores que han referido correlaciones moderadas del agotamiento emocional con sintomatología somática, evidenciando que esta dimensión del burnout es la que más se asocia a la salud física y sus efectos fisiológicos son muy semejantes a los asociados al estrés o la fatiga crónicos (Esteras et al., 2018; Grossi et al., 2003; Maslach, Schaufeli y Leiter, 2001; Sandín, 2008).

Cabría asumir que el agotamiento emocional podría asociarse al estrés reciente y crónico (Sandín, 2008; Sandín y Chorot, 2017) y/o a pérdida de motivación del individuo (Campagne, 2012) y generar vulnerabilidad para el desarrollo de enfermedades físicas. Nuestros datos están en línea con el grueso de la literatura científica sobre el tema; por ejemplo, se ha constatado que las alteraciones psicosomáticas se asocian con el agotamiento profesional crónico (Pando et al., 2006). Así mismo, el agotamiento emocional parece tener ciertas similitudes con el denominado «agotamiento vital», el cual se ha asociado de forma significativa a los trastornos emocionales (especialmente depresión) y psicosomáticos (en particular con la cardiopatía coronaria) (véase Sandín, 2002, 2008). El agotamiento emocional se ha vinculado de forma específica al estrés laboral, incluyendo la baja satisfacción laboral, y al estrés de rol en el personal docente (Esteras, Chorot y Sandín, 2014). En una línea similar a lo indicado por Honkonen et al. (2006), encontramos que la sintomatología física de tipo muscu- lo-esquelético se asocia significativamente a las tres dimensiones de burnout. Este resultado es consistente con la idea de que cualquiera de las tres dimensiones de burnout posee una relación estrecha con los estados de tensión psicológica, incluidas las manifestaciones de tensión muscular, como aspecto genérico asociado al burnout.

Por otra parte, nuestros resultados apoyan los datos obtenidos por Gil-Monte, Núñez y Selva (2006) en una muestra de profesionales de enfermería, los cuales encontraron una relación positiva y significativa entre el síndrome de burnout y la percepción subjetiva de alteraciones de tipo cardiovascular. Constataron que la correlación más intensa se daba para los niveles de agotamiento emocional y la menos intensa para la eficacia profesional. En el presente estudio encontramos una relación significativa entre las dimensiones de agotamiento emocional y despersonalización con la patología de tipo cardiovascular y otras manifestaciones de sintomatología física, incluidos los síntomas de tipo inmunológico. Estos resultados son congruentes con los obtenidos en trabajos precedentes, como los referidos por Nakamura, Nagase, Yoshida y Ogino (1999), los cuales encontraron que la despersonalización se asociaba negativamente a los niveles de células NK (tipo linfocito perteneciente al sistema inmunitario), por lo que la despersonalización se asociaba a mayor vulnerabilidad del sistema inmunológico.

Los datos obtenidos en relación con los síntomas psicopatológicos proporcionan información similar a la encontrada para los síntomas físicos, i.e., la principal asociación de estos síntomas se da con la dimensión de 
agotamiento emocional, y en un grado notablemente menor con las dos restantes dimensiones (despersonalización y realización personal). Estos resultados son consistentes con el grueso de la literatura que indica una mayor implicación de la sintomatología psicopatológica con la variante del burnout de agotamiento emocional, especialmente la sintomatología referida a los síntomas de tipo neurótico (somatización, depresión, ansiedad, obsesión-compulsión, etc.) (Aluja, 1997; Calvete y Vi1la, 2000). Mención aparte merece la particular asociación que parece darse entre los síntomas de ideación paranoide y las dimensiones de burnout, ya que correlacionó significativamente con las tres escalas de burnout; así como también la correlación significativa entre los síntomas psicóticos y la dimensión de despersonalización (véase la Tabla 2). Estos resultados apoyan la evidencia preliminar referida por Cáceres (2006) sobre la asociación entre la despersonalización y los síntomas de ideación paranoide y personalidad psicótica en profesionales de la sanidad.

En relación con el segundo objetivo del estudio, esperábamos encontrar diferencias significativas entre los individuos con diagnóstico de síndrome de burnout y los individuos sin el síndrome, para las diferentes variables de sintomatología física y mental. A este respecto, encontramos puntuaciones medias significativamente superiores asociadas al síndrome de burnout para la mayor parte de las dimensiones sintomatológicas, incluyendo las correspondientes a los sistemas inmunológico general, cardiovascular, respiratorio, neurosensorial, músculo-esquelético, y puntuación global en síntomas somáticos. Los resultados están en coherencia con trabajos precedentes que confirman que el síndrome de burnout aparece asociado a trastornos físicos heterogéneos, incluidos los distintos tipos de dolor y molestias físicas, y las alteraciones de índole cardiovascular, respiratorio, inmunológico e infeccioso (Bargellini et al., 2000; Freudenberger y Richelson, 1980; García, 1991; Golembiewski, Munzenrider, Stevenson, 1986; Maslach y Jackson, 1981).

En una línea similar, constatamos una estrecha relación entre el síndrome de burnout y la sintomatología psicopatológica, lo cual también proporciona apoyo empírico a nuestra segunda hipótesis. Salvo para algunos tipos de síntomas (hostilidad, sensibilidad interpersonal, ansiedad fóbica y psicoticismo), encontramos diferencias significativas en síntomas psicopatológicos entre los docentes con síndrome de burnout y los docentes libres del síndrome, siendo siempre más elevados los síntomas en el grupo de participantes con diagnóstico del síndrome de burnout. Salvo el efecto significativo observado para los síntomas de ideación paranoide, los tipos de sín- tomas más estrechamente vinculados al síndrome de burnout corresponden a síntomas de tipo neurótico, incluyendo los síntomas de ansiedad, depresión, obsesión-compulsión y somatización. Estos datos confirman y amplían al ámbito de los profesionales de la enseñanza la evidencia previamente constatada para esta y otras áreas, especialmente para campo de los profesionales de la salud (p.ej., García et al., 2000; Firth et al., 1987; Gómez-Alcaina et al., 2013; Revicky et al., 1991; Silva, Bolsoni-Silva, y Loureiro, 2018).

En suma, hemos encontrado una estrecha asociación entre una de las dimensiones del burnout, i.e., el agotamiento emocional, y los síntomas somáticos y psicopatológicos. Tal asociación parece ser inespecífica ya que todos los tipos de síntomas (excepto los de psicoticismo) correlacionaron de forma significativa con el agotamiento emocional. Es probable que esta dimensión posea cierta semejanza y/o solapamiento con el concepto de «agotamiento vital» (véase Sandín, 2002, 2008), el cual también se ha relacionado de forma genérica con la sintomatología física y emocional. Las dos restantes dimensiones del burnout presentan un menor grado de asociación con la sintomatología somática y psicopatológica, aunque sí parecen tener cierta asociación específica con algunos síntomas. Por ejemplo, la despersonalización, se relaciona significativamente con los síntomas de tipo neurosensorial, y con la ideación paranoide y los síntomas psicóticos; mientras que la dimensión de realización personal se relaciona más específicamente con la ansiedad, la depresión y la tensión muscular. Estos patrones de correlación son coherentes con el significado conceptual de ambas dimensiones del burnout.

Aunque el estudio proporciona nueva información relevante para conocer las características psicosomáticas y psicopatológicas asociadas a las dimensiones del burnout y al síndrome de burnout, el trabajo posee varias limitaciones. Una primera limitación consiste en que la información procede en su totalidad de pruebas de autoinforme. Otras fuentes de información, tales como las procedentes de estimaciones clínicas y entrevistas, y/o de evaluación psicofisiológica o comportamental, podrían proporcionar información adicional para un mejor conocimiento de los perfiles psicopatológicos asociados al burnout. Una segunda limitación viene dada por haber utilizado un diseño de tipo correlacional. Este tipo de diseño no permite establecer relaciones causales entre el burnout y los síntomas psicosomáticos y psicopatológicos, y por tanto no nos posibilita saber si los síntomas son consecuencia o causa del burnout. Futuras investigaciones deberán abordar esta cuestión mediante diseños longitudinales, y contrastarlo y extenderlo a otras profe- 
siones asociadas al burnout en orden a implementar terapias y estrategias preventivas eficaces.

\section{Conflictos de intereses}

Los autores declaran que no existen conflictos de intereses

\section{Referencias}

Aluja, A. (1997). Burnout profesional en maestros y su relación con indicadores de salud mental. Boletín de Psicología, 55, 47-61.

Ballester-Arnal, R., Gómez-Martínez, S., Gil-Juliá, B., Ferrándiz-Sellés, M. D., \& Collado-Boira, E. J. (2016). Burnout y factores estresantes en profesionales sanitarios de las unidades de cuidados intensivos. Revista de Psicopatología y Psicología Clínica, 21, 129-136.

Bargellini, A., Barbieri A., Rovesti S., Vivoli, R., Roncaglia, R., \& Borella, P. (2000). Relation between immune variables and burnout in a simple of physicians. Occupational and Environmental Medicine, 57, 453-457.

Cáceres, G. (2006). Prevalencia del síndrome de burnout en personal sanitario militar. (Tesis doctoral). Universidad Complutense de Madrid.

Calvete, E., y Villa, A. (2000). Burnout y síntomas psicológicos: modelo de medida y relaciones estructurales. Ansiedad y Estrés 6, 117-130.

Campagne, D. M. (2012). When therapist run out of steam: Professional boredom or burnout? Revista de Psicopatología y Psicología Clínica, 17, 75-85.

Capone, V., Joshanloo, M., \& Park, M. S. (2019). Burnout, depression, efficacy beliefs, and work-related variables among school teachers. International Journal of Educational Research. Disponible electrónicamente en: https://doi.org/10.1016/j.ijer.2019.02.001.

Esteras, J., Chorot, P., y Sandín, B. (2018). Es síndrome de burnout en los docentes. Pirámide: Madrid.

Esteras, J., Chorot, P., y Sandín, B. (2014). Predicción de los niveles de burnout en los docentes: Factores protectores y de vulnerabilidad. Revista de Psicopatología y Psicología Clínica, 19, 79-92.

Durán, M. A., Extremera, N., Montalbán, F., y Rey, L. (2005). Engagement y Burnout en el ámbito docente: Análisis de sus relaciones con la satisfacción laboral y vital en una muestra de profesores. Revista de Psicología del Trabajo y de las Organizaciones, 21, 145-158.

Fernández, G. (2000). Burnout y trastornos psicosomáticos. Revista de psicología del trabajo y de las organizaciones, 16 , 229-235

Firth, H., McIntee, J., McKeown, P., \& Britton, P. (1987): Professional depression, «burnout» and personality in longstay nursing. International Journal of Nursing Studies, 24, 227-237.

Freudenberger, H. J. \& Richelson, G. (1980). Burnout: The high cost of high achievement. Nueva York: Anchor Press.

García, M. (1991). Burnout en profesionales de enfermería de centros hospitalarios. Revista de Psicología del Trabajo y de las Organizaciones, 7, 3-12.
García, M., Sáez, M. C., y Llor, B. (2000). Burnout, satisfacción laboral y bienestar en personal sanitario de salud mental. Revista de Psicología del Trabajo y de las Organizaciones, 16 , 215-228

Gil-Monte, P. R., Valcáercel, P., Grau, R. M. y Peiró, J. M. (1996). La incidencia del síndrome de burnout sobre la salud: un estudio correlacional en profesionales de enfermería. Psiquis, 17, 37-42.

Gil-Monte, P. R., Núñez, E. y Selva, Y. (2006). Relación entre el síndrome de quemarse por el trabajo (burnout) y síntomas cardiovasculares: un estudio en técnicos de prevención de riesgos laborales. Interamerican Journal of Psychology, 40, 227-232.

Golembiewski, R. T. \& Munzenrider, R. F. \& Stevenson, J. (1986). Stress in organizations. New York: Praeger.

Gómez-Alcaina, B., Montero-Marín, J., Demarzo, M. M., Pereira, J. P., \& García-Campayo, J. (2013). Utilidad de los marcadores biológicos en la detección precoz y prevención del síndrome de burnout. Revista de Psicopatología y Psicología Clínica, 18, 245-253.

Greenglass, E., \& Burke, R. (1988). Work and family precursors of burnout in teachers: Sex differences. Sex Roles, 18, 215229.

Grossi, G., Perski, A., Evengard, B., Blomkvist V., \& Orth-Gomér, K. (2003). Physiological correlates of burnout among women. Journal of Psychosomatic Research, 55, 309-316.

Guglielmi, R. S. \& Tatrow, D. (1998). Occupational stress burnout and health in teachers: A methodological and theoretical analysis. Review of Educational Reseanh, 68, 61-100.

Haack, M.R. (1988). Stress and impairment among nursing students. Research in Nursing and Health, 11, 125-134.

Holt, P., Fine, M., \& Tollefson, N. (1987). Mediating Stress: survival of the hardy. Psychology in the Schools, 24, 51-58.

Honkonen, T., Ahola, K., Pertovaara, M., Isometä, E., Kalimo, R., Nykyri, E., Aromaa, A. \& Lönnqvist, J. (2006). The association between burnout and physical illness in the general population- Results fron the Finis Health 2000 Study. Journal of Psychosomatic Resarch, 6, 59-66.

Maslach, C. \& Jackson, S.E. (1981): Maslach Burnout Inventory. Palo Alto, CA; University of California: Consulting Psychologists Press.

Maslach, C., Schaufeli, W. B. \& Leiter, M. P. (2001). Job burnout. Annual Review of Psychology, 52, 397-422.

Montero-Marín, J., Prado-Abril, J., Demarzo, M. M. P., García-Toro, M., \& García-Campayo, J. (2016). Burnout subtypes and their clinical implications: A theoretical proposal for specific therapeutic approaches. Revista de Psicopatología y Psicología Clínica, 2, 231-242.

Moya-Albiol, L., Serrano, M. A., González-Bono, E., Rodríguez-Alarcón, G. y Salvador, A. (2005): Respuesta psicofisiológica de estrés en una jornada laboral. Psicothema, 17, 205211.

Nakamura, H., Nagase, H., Yoshida, M., \& Ogino, K. (1999). Natural Killer (NK) cell activity and NK cell subsets in workers with a tendency of Burnout. Journal of Psychosomatic Research, 46, 569-578.

Pando, M., Aranda, C., Aldrete, M. G., Flores, E., y Pozos, E. (2006). Factores Psicosociales y burnout en docentes del Centro Universitario de Ciencias de la Salud. Investigación en Salud. 8, 173-177. 
Revicki, D., May, H., \& Whitley, T. (1991). Reliability and validity of the Work related Strain Inventory among health professionals. Behavioral Medicine, 17, 111-120.

Sandín, B. (2002). Papel de las emociones negativas en el trastorno cardiovascular: Un análisis crítico. Revista de Psicopatología y Psicología Clínica, 7, 1-18.

Sandín, B. (2008). El estrés psicosocial (2 ${ }^{\mathrm{a}}$ ed.). Madrid: Klinik.

Sandín, B., y Chorot, P. (2017). Cuestionario de Sucesos Vitales (CSV): Estructura factorial, propiedades psicométricas y datos normativos. Revista de Psicopatología y Psicología Clínica, 22, 95-115.

Sandín, B., Valiente, R. M., y Chorot, P. (2008). Instrumentos para la evaluación del estrés psicosocial. En B. Sandín (Ed.), El estrés psicosocial: Conceptos y consecuencias clínicas $\left(2^{\mathrm{a}}\right.$ ed., pp. 267-346). Madrid: Klink.

Sandín, B., Valiente, R. M., Chorot, P., Santed, M. A., y Lostao, L. (2008). SA-45: forma abreviada del SCL-90. Psicothema, 20, 290-296.

Santamaría, M. I., y Soto, R. (2005). Prevalencia del burnout en la enfermería de atención primaria. Enfermería Clínica, 16, 123130 .

Seisdedos, N. (1997). Manual MBI: Inventario burnout de Maslach. Madrid: TEA Ediciones.

Silva, N. R., Bolsoni-Silva, A. T., y Loureiro, S. R. (2018). Burnout syndrome and depression in elementary school teachers: A correlational study. Revista Brasileira de Educacao, 23, e230048. https://doi.org/10.1590/S1413-24782018230048. 
